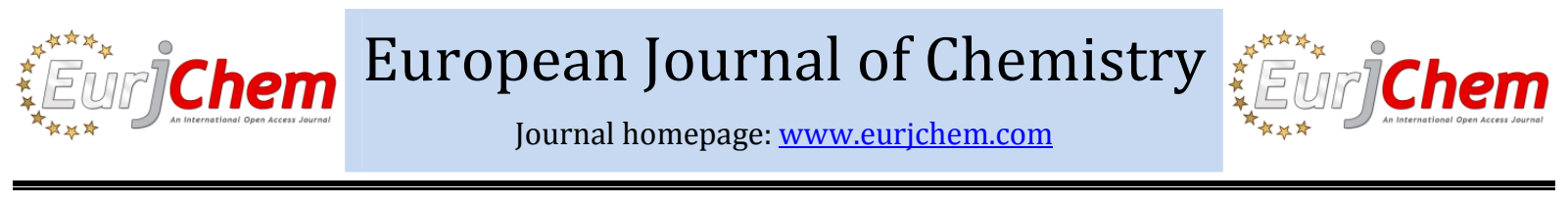

\title{
Synthesis and evaluation of antimitotic activity of new tetralone acid analogues of podophyllotoxin
}

\author{
Santhekasalagere Basavaiah Shivakumar, Yeriyur Basavaiah Basavaraju *, \\ Basavaiah Umesha, Mudeenahally Hucchegowda Krishna and Ningegowda Mallesha \\ Department of Studies in Chemistry, University of Mysore, Manasagangotri, Mysore, 570006, Karnataka, India \\ ${ }^{*}$ Corresponding author at: Department of Studies in Chemistry, University of Mysore, Manasagangotri, Mysore, 570006, Karnataka, India. \\ Tel.: +91.948.0441804. Fax: +91.821.2421263. E-mail address: basavaraju yb@yahoo.co.in (Y.B. Basavaraju).
}

\section{ARTICLE INFORMATION}

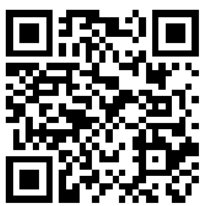

DOI: 10.5155 /eurjchem.5.3.424-429.1020

Received: 20 January 2014

Received in revised form: 23 March 2014

Accepted: 25 March 2014

Online: 30 September 2014

\section{KEYWORDS}

\section{Ketones}

Itaconic acids

Tetralone acids

Antimitotic activity

Stobbe condensation

Catalytic hydrogenation

\section{Introduction}

Podophyllotoxin [1] (Figure 1) and its several analogues are being used as cytotoxic spindle poisons and antitumor agents at clinical levels [2]. Several analogues of the podophyllotoxin have been reported in literature. Podophyllotoxin has been extracted from two important medicinal plants named podophyllum emodi an Indian species and podophyllum peltatum, a North American species [3]. It also occurs in many other plants of podophyllum species. It belongs to the family of natural products called lignans. Podophyllotoxin showed other biological activities such as cathartic, antitropical skin disease, antimalarial, anti-HIV (AIDS) etc. [4].

In view of the above facts, it was decided to modify the structure of podophyllotoxin and synthesized tetralone acids as analogues [5-8]. They were synthesized by replacing 3,4,5trimethoxyphenyl ring with cyclohexyl group in podophyllotoxin and 1,3-methylene dioxy ring with methoxy, methyl, thiomethyl group and hydrogen and fluorine elements and lactone ring with carboxylic acid group. The analogues of podophyllotoxin were synthesized using Gensler's method [9] with some changes in reagents and experimental procedure. The synthesized tetralone acids were screened for their antimitotic activity by onion root method [10].

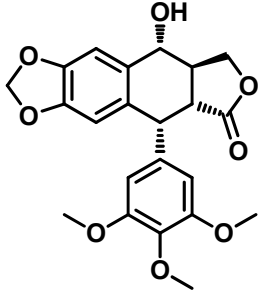

Figure 1. The structure of podophyllotoxin.

\section{Experimental}

\subsection{Materials and methods}

All the reagents and chemicals were purchased. They were used without further purification. Melting points were taken in open capillary tubes and are uncorrected. Thin layer chromatography (TLC) is performed with E. Merck pre-coated silica gel plates $\left(60 \mathrm{~F}_{254}\right)$. Acme, India silica gel, $60-120$ mesh is used for column chromatography. IR spectra in $\mathrm{KBr}$ were recorded on Perkin-Elmer model 683 spectrometers. 


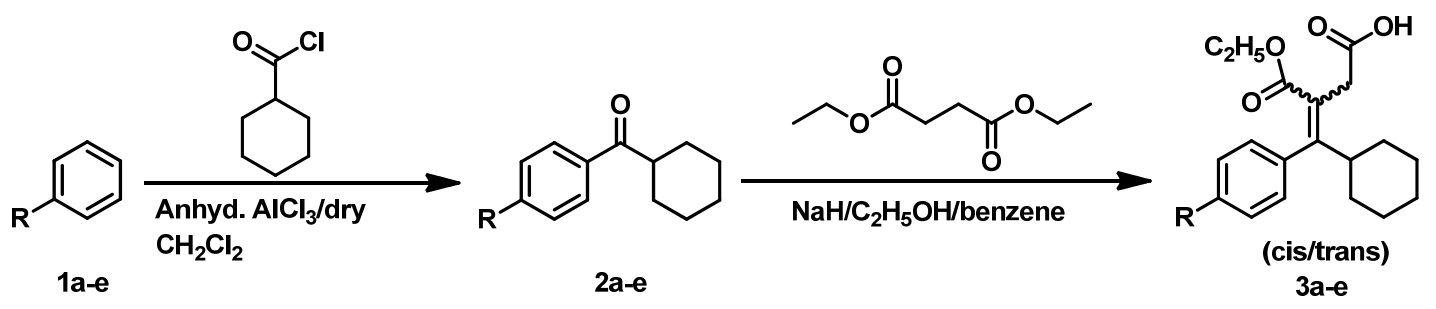<smiles>[R]c1ccc(C(C2CCCCC2)C2CC(=O)OC2=O)cc1</smiles>

6a-e<smiles>[R]c1ccc2c(c1)C(=O)C[C@H](C(=O)O)[C@H]2C1CCCCC1</smiles>

$7 a-e$

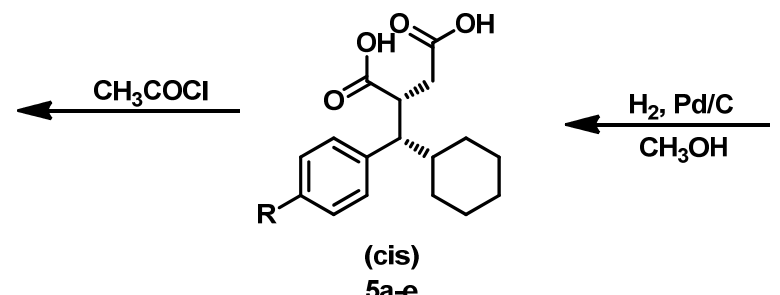

5a-e<smiles>CC(CO)C(O)O[N+](=O)[O-]</smiles>

R: $\mathbf{a}=\mathrm{OCH}_{3}$

$\mathbf{b}=\mathbf{H}$

$\mathrm{c}=\mathrm{CH}_{3}$

$\mathrm{d}=\mathrm{SCH}_{3}$

$\mathbf{e}=\mathbf{F}$

Scheme 1

${ }^{1} \mathrm{H}$ NMR (400 MHz) and ${ }^{13} \mathrm{C}$ NMR (100 MHz) spectra were recorded using tetramethyl silane (TMS) as an internal reference on Bruker spectrometer. Elemental analyses were performed on a Perkin-Elmer 2400. Mass spectra were obtained by Water-Q-TOF ultima spectrometer. Micro analytical data were obtained by Elemental-Vario EL-III.

\subsection{Synthesis}

\subsubsection{Procedure for the preparation of substituted cyclohexyl(phenyl)methanone (2a-e)}

A solution of cyclohexane carbonyl chloride $(16.2 \mathrm{~g}, 0.111$ $\mathrm{mol}$ ) in dichloromethane $(50 \mathrm{~mL})$ was added drop wise to the stirred suspension of substituted benzene (1a-e) $(10 \mathrm{~g}, 0.092$ $\mathrm{mol}$ ) and anhydrous aluminium chloride ( $13.48 \mathrm{~g}, 0.101 \mathrm{~mol})$ in dichloromethane $(50 \mathrm{~mL})$ at $0-5{ }^{\circ} \mathrm{C}$ under nitrogen gas atmosphere. The reaction mixture was stirred for $5 \mathrm{~h}$ at 25-30 ${ }^{\circ} \mathrm{C}$. The completion of the reaction was known by TLC. The reaction mass was poured into $1 \mathrm{~N} \mathrm{HCl}$. The mixture was stirred for $2 \mathrm{~h}$ at $25-30{ }^{\circ} \mathrm{C}$. The product was extracted into dichloromethane. The combined organic layer was washed with aq. sodium bicarbonate solution and then with water, dried over anhydrous $\mathrm{Na}_{2} \mathrm{SO}_{4}$. The organic layer was concentrated under vacuum using rotary evaporator. The products were recrystallized from diethyl ether (Scheme 1).

Cyclohexyl(4-methoxyphenyl)methanone (2a): Color: White solid. Yield: $74.40 \%$. M.p.: $61-63{ }^{\circ} \mathrm{C}$. IR $\left(\mathrm{KBr}, v, \mathrm{~cm}^{-1}\right): 2926$ (CH), $2973($ Ar-H), 1738 (ester, $\mathrm{C}=0), 1697(\mathrm{C}=0) .{ }^{1} \mathrm{H}$ NMR (400 $\left.\mathrm{MHz}, \mathrm{CDCl}_{3}, \delta, \mathrm{ppm}\right): 1.20-1.80\left(\mathrm{~m}, 10 \mathrm{H}\right.$, cyclic $\left.-\mathrm{CH}_{2}\right), 2.23-2.65$ (p, 1H, cyclic- $\mathrm{CH}), 3.78$ (s, 3H, OCH 3 ), 6.90-7.90 (m, 4H, Ar-H). ${ }^{13} \mathrm{C}$ NMR $\left(100 \mathrm{MHz}, \mathrm{CDCl}_{3}, \delta, \mathrm{ppm}\right): 25.1,25.6,25.9,29.1,29.6$, 47.3, 55.7, 114.1, 114.5, 129, 129.2, 129.4, 165.4, 202.3. MS (ESI, $\mathrm{m} / \mathrm{z}$ ): $219.10\left(\mathrm{M}^{+}\right)$. Anal. calcd. for $\mathrm{C}_{14} \mathrm{H}_{18} \mathrm{O}_{2}$ : C, 77.03; $\mathrm{H}$, 8.31. Found: $\mathrm{C}, 77.01 ; \mathrm{H}, 8.27 \%$.

Cyclohexyl(phenyl)methanone (2b): Color: White crystalline solid. Yield: $71.91 \%$. M.p.: $52-54{ }^{\circ} \mathrm{C}$. IR $\left(\mathrm{KBr}, v, \mathrm{~cm}^{-1}\right): 1685$ $(\mathrm{C}=0), 2995$ (Ar CH). ${ }^{1} \mathrm{H}$ NMR $\left(400 \mathrm{MHz}, \mathrm{CDCl}_{3}, \delta, \mathrm{ppm}\right): 2.10$ $1.23\left(\mathrm{~m}, 10 \mathrm{H}\right.$, cyclic $\left.-\mathrm{CH}_{2}\right), 2.14-2.68(\mathrm{p}, 1 \mathrm{H}$, cyclic $-\mathrm{CH}), 6.70-$ $7.40(\mathrm{~m}, 5 \mathrm{H}, \mathrm{Ar}-\mathrm{H}) .{ }^{13} \mathrm{C}$ NMR (100 MHz, $\left.\mathrm{CDCl}_{3}, \delta, \mathrm{ppm}\right): 25.0$, 25.3, 25.7, 29.2, 29.7, 47.9, 128, 128.2, 128.4, 128.9, 133.4 139.4, 220.5. MS (ESI, $m / z)$ : $189.06\left(\mathrm{M}^{+}\right)$. Anal. calcd. for $\mathrm{C}_{13} \mathrm{H}_{16} \mathrm{O}$ : C, 82.94; $\mathrm{H}, 8.57$. Found: $\mathrm{C}, 82.91 ; \mathrm{H}, 8.54 \%$.

Cyclohexyl(p-tolyl)methanone (2c): Color: White solid. Yield: 70.15 \%. M.p.: $71-73{ }^{\circ} \mathrm{C}$. IR (KBr, v, $\left.\mathrm{cm}^{-1}\right): 1696$ (C=0), 2927 (Methyl CH ), 3030 (Ar CH). ${ }^{1} \mathrm{H}$ NMR $\left(400 \mathrm{MHz}, \mathrm{CDCl}_{3}, \delta\right.$, ppm): 0.93-1.93 (m, 10H, cyclic - $\left.\mathrm{CH}_{2}\right), 2.38\left(\mathrm{~s}, 3 \mathrm{H},-\mathrm{CH}_{3}\right), 2.64$ (p, 1H, cyclic -CH), 6.82-7.37 (m, 4H, Ar-H). ${ }^{13} \mathrm{C}$ NMR $(100 \mathrm{MHz}$, $\left.\mathrm{CDCl}_{3}, \delta, \mathrm{ppm}\right): 21.9,25.1,25.6,25.7,29.1,29.3,47.8,128.1$, 
128.4, 128.7, 128.9, 133.5, 142.6, 202.5. MS (ESI, $m / z): 203.10$ $\left(\mathrm{M}^{+}\right)$. Anal. calcd. for $\mathrm{C}_{14} \mathrm{H}_{18} \mathrm{O}: \mathrm{C}, 83.12 ; \mathrm{H}$, 8.97. Found: C, 83.09; $\mathrm{H}, 8.95 \%$.

Cyclohexyl(4-(methylthio)phenyl)methanone (2d): Color: White solid. Yield: $75.05 \%$. M.p.: 87-89 ${ }^{\circ} \mathrm{C}$. IR $\left(\mathrm{KBr}, v, \mathrm{~cm}^{-1}\right)$ : $1706(\mathrm{C}=0)$. ${ }^{1} \mathrm{H}$ NMR $\left(400 \mathrm{MHz}, \mathrm{CDCl}_{3}, \delta, \mathrm{ppm}\right): 1.44-1.80(\mathrm{~m}$, $10 \mathrm{H}$, cyclic $\left.-\mathrm{CH}_{2}\right), 2.56(\mathrm{p}, 1 \mathrm{H}$, cyclic $-\mathrm{CH}), 2.54\left(\mathrm{~s}, 3 \mathrm{H},-\mathrm{SCH}_{3}\right)$, 6.70-7.26 (m, 4H, Ar-H). ${ }^{13} \mathrm{C}$ NMR (100 MHz, $\left.\mathrm{CDCl}_{3}, \delta, \mathrm{ppm}\right):$ $14.7,25.2,25.5,25.8,29.4,29.7,47.9,126.4,126.9,132.1$, 132.6, 133.4, 143.5, 202.4. MS (ESI, $m / z): 235.02\left(M^{+}\right)$. Anal. calcd. for $\mathrm{C}_{14} \mathrm{H}_{18} \mathrm{OS}$ : C, 71.75; H, 7.74. Found: $\mathrm{C}, 71.73 ; \mathrm{H}, 7.70 \%$.

Cyclohexyl(4-fluorophenyl)methanone (2e): Color: White gummy mass. Yield: $70.21 \%$. IR $\left(\mathrm{KBr}, \mathrm{v}, \mathrm{cm}^{-1}\right): 1690(\mathrm{C}=0) .{ }^{1} \mathrm{H}$ NMR (400 MHz, $\left.\mathrm{CDCl}_{3}, \delta, \mathrm{ppm}\right): 1.13-1.87\left(\mathrm{~m}, 10 \mathrm{H}\right.$, cyclic $\left.-\mathrm{CH}_{2}\right)$, 2.76 (p, $1 \mathrm{H}$, cyclic -CH), 7.13-7.74 (m, 4H, Ar-H). ${ }^{13} \mathrm{C}$ NMR $(100$ $\left.\mathrm{MHz}, \mathrm{CDCl}_{3}, \delta, \mathrm{ppm}\right): 25,25.3,25.9,29.2,29.5,47.9,115.4$, $115.7,130.2,130.7,132.9,167.7,202.8$. MS (ESI, $m / z): 206.08$ $\left(\mathrm{M}^{+}\right)$. Anal. calcd. for $\mathrm{C}_{13} \mathrm{H}_{15} \mathrm{FO}$ : $\mathrm{C}, 75.70$; $\mathrm{H}, 7.33$. Found: $\mathrm{C}$, 75.68; H, 7.30\%.

\subsubsection{General procedure for the synthesis of substituted 2-(cyclohexyl(phenyl)methylene)succinic acid (4a-e)}

Sodium hydride $(2.47 \mathrm{~g}, 0.103 \mathrm{~mol}$ ) was added in portions to the stirred suspension of substituted cyclohexyl(phenyl) methanone (2a-e) $(5 \mathrm{~g}, 0.0229 \mathrm{~mol})$ and diethyl succinate (11.9 $\mathrm{g}, 0.068 \mathrm{~mol})$ in dry benzene $(15 \mathrm{~mL})$ under nitrogen gas atmosphere. The reaction mixture was stirred for $10 \mathrm{~min}$ at 25$30{ }^{\circ} \mathrm{C}$ (until the evolution of hydrogen gas ceased). Absolute ethanol (5.2 g, $0.114 \mathrm{~mol}$ ) was added drop wise during $1 \mathrm{~h}$ to the above solution. The reaction mass was stirred at $25-30{ }^{\circ} \mathrm{C}$ for $15 \mathrm{~h}$. The completion of the reaction was confirmed by TLC. The reaction mixture was cooled and acidified by addition of glacial acetic acid. The substituted succinic half esters were extracted into ether and then into saturated sodium carbonate solution. The sodium carbonate extract was neutralized by dilute acetic acid. The precipitated gummy residue was extracted into dichloromethane. The organic layer was washed with water and concentrated under vacuum using rotary evaporator to give white solid in good yields. The substituted succinic acid half esters (3a-e) ( $4 \mathrm{~g}, 0.0115 \mathrm{~mol}$ ) were added to the stirred solution of methanol $(15 \mathrm{~mL})$, and sodium hydroxide solution $(15 \mathrm{~mL})(2.3 \mathrm{~g}, 0.057 \mathrm{~mol})$. The resultant reaction mixture was refluxed at $75-80^{\circ} \mathrm{C}$ for $5 \mathrm{~h}$. Here again the completion of the reaction was assessed with TLC. The reaction mixture was concentrated to give slight yellow residue. The resultant residue was dissolved with $40 \mathrm{~mL}$ water. The aqueous layer was neutralized with dilute hydrochloric acid to obtain gummy light brownish mass and extracted to dichloromethane. The separated organic layer washed with water, dried over anhydrous sodium sulphate and concentrated under reduced pressure. The residue was dissolved in $30 \mathrm{~mL}$ diethyl ether and kept overnight in fridge. The products precipitated as white needle type crystals and were filtered to afford pure compounds in good yields (Scheme 1).

2-(Cyclohexyl(4-methoxyphenyl)methylene)succinic acid (4a): Color: white crystal solid. Yield: 88.10\%. M.p.: 165-167 ${ }^{\circ} \mathrm{C}$. IR (KBr, v, cm-1): 3500 (carboxylic $\left.\mathrm{OH}\right), 1713.8\left(\mathrm{CH}_{2}-\mathrm{C}=0\right)$, $1663(\alpha, \beta$ unsaturated $\mathrm{C}=0), 1605$ (Ar $\mathrm{C}=\mathrm{C}) .{ }^{1} \mathrm{H}$ NMR $(400$ $\left.\mathrm{MHz}, \mathrm{CDCl}_{3}, \delta, \mathrm{ppm}\right): 1.14-1.62\left(\mathrm{~m}, 10 \mathrm{H}\right.$, cyclic $\left.-\mathrm{CH}_{2}\right), 2.40(\mathrm{p}, 1$ $\mathrm{H}$, cyclic $-\mathrm{CH}), 3.62\left(\mathrm{~s}, 2 \mathrm{H},-\mathrm{CH}_{2}\right), 3.78\left(\mathrm{~s}, 3 \mathrm{H},-\mathrm{OCH}_{3}\right), 6.90-7.33$ (m, 4H, Ar-H). ${ }^{13} \mathrm{C}$ NMR (100 MHz, $\left.\mathrm{CDCl}_{3}, \delta, \mathrm{ppm}\right): 25.1,25.7,26$, $26.2,26.5,32.5,34.8,55.5,114,117.5,129.5,129.8,131.4$, 155.1, 159.8, 171.6, 174.4. MS (ESI, $m / z): 318.11\left(\mathrm{M}^{+}\right)$. Anal. calcd. for $\mathrm{C}_{18} \mathrm{H}_{22} \mathrm{O}_{5}$ : C, 67.91; $\mathrm{H}, 6.97$. Found: $\mathrm{C}, 67.89 ; \mathrm{H}, 6.94 \%$.

2-(Cyclohexyl(phenyl)methylene)succinic acid (4b): Color: Light yellow solid. Yield: 78.05 \%. M.p.: $159-161{ }^{\circ} \mathrm{C}$. IR (KBr, $v$, $\mathrm{cm}^{-1}$ ): 3400-3200 (carboxylic OH), $1603(\mathrm{Ar} \mathrm{C}=\mathrm{C}), 1701\left(\mathrm{CH}_{2^{-}}\right.$ $\mathrm{C}=0), 1660(\alpha, \beta$ unsaturated $\mathrm{C}=0) .{ }^{1} \mathrm{H}$ NMR $\left(400 \mathrm{MHz}, \mathrm{CDCl}_{3}, \delta\right.$, ppm): 0.89-1.63 (m, 10H, cyclic - $\left.\mathrm{CH}_{2}\right), 2.36(\mathrm{p}, 1 \mathrm{H}$, cyclic $-\mathrm{CH})$, $3.70\left(\mathrm{~s}, 2 \mathrm{H},-\mathrm{CH}_{2}\right), 6.84-7.3(\mathrm{~m}, 5 \mathrm{H}, \mathrm{Ar}-\mathrm{H}) .{ }^{13} \mathrm{C}$ NMR $(100 \mathrm{MHz}$,
$\left.\mathrm{CDCl}_{3}, \delta, \mathrm{ppm}\right): 25.4,25.7,26,26.1,26.4,32.9,34.4,117.7$, $126.2,126.9,127.8,128.4,128.8,139.4,155.8,171.9,174.4$. MS (ESI, $m / z$ ): $289.13\left(\mathrm{M}^{+}\right.$). Anal. calcd. for $\mathrm{C}_{17} \mathrm{H}_{20} \mathrm{O}_{4}: \mathrm{C}, 70.81 ; \mathrm{H}$, 6.99. Found: C, 70.78; $\mathrm{H}, 6.95 \%$.

2-(Cyclohexyl(p-tolyl)methylene)succinic acid (4c): Color: Light yellow solid. Yield: 80.15 \%. M.p.: $172-174{ }^{\circ} \mathrm{C}$. IR (KBr, $v$, $\mathrm{cm}^{-1}$ ): 3500 (cyclic OH), 1600 (Ar C=C), $1680\left(\mathrm{CH}_{2}-\mathrm{C}=0\right.$ ), 1664 $(\alpha, \beta$ unsaturated $\mathrm{C}=0) .{ }^{1} \mathrm{H}$ NMR $\left(400 \mathrm{MHz}, \mathrm{CDCl}_{3}, \delta, \mathrm{ppm}\right)$ : 0.96-1.55 (m, 10H, cyclic - $\left.\mathrm{CH}_{2}\right), 2.36-2.54(\mathrm{p}, 1 \mathrm{H}$, cyclic $-\mathrm{CH})$, 2.56 (s, 3H, $\left.-\mathrm{CH}_{3}\right), 3.62$ (s, $\left.2 \mathrm{H},-\mathrm{CH}_{2}\right), 7.10-7.59$ (m, 4H, Ar-H). ${ }^{13} \mathrm{C}$ NMR $\left(100 \mathrm{MHz}, \mathrm{CDCl}_{3}, \delta\right.$, ppm): 21.7, 25.1, 25.8, 26.1, 26.4, 26.7, 32.1, 34.7, 117.7, 126.2, 126.7, 128.4, 128.9, 136.1, 137.4, 155.1, 171.4, 174.2. MS (ESI, $m / z): 303.15\left(\mathrm{M}^{+}\right)$. Anal. calcd. for $\mathrm{C}_{18} \mathrm{H}_{22} \mathrm{O}_{4}$ : C, 71.50; $\mathrm{H}, 7.33$. Found: $\mathrm{C}, 71.49$; $\mathrm{H}, 7.31 \%$.

2-(Cyclohexyl(4-(methylthio)phenyl)methylene)succinic acid (4d): Color: Light yellow solid. Yield: $92.61 \%$. M.p.: $147-149^{\circ} \mathrm{C}$. IR ( $\left.\mathrm{KBr}, v, \mathrm{~cm}^{-1}\right)$ : 3435 (carboxylic $\left.\mathrm{OH}\right), 1685\left(\mathrm{CH}_{2}-\mathrm{C}=0\right), 1663$ $(\alpha, \beta$ unsaturated $\mathrm{C}=0) .{ }^{1} \mathrm{H}$ NMR $\left(400 \mathrm{MHz}, \mathrm{CDCl}_{3}, \delta, \mathrm{ppm}\right)$ : 1.13-1.65 (m, 10H, cyclic - $\left.\mathrm{CH}_{2}\right), 2.10(\mathrm{p}, 1 \mathrm{H}$, cyclic $-\mathrm{CH}), 2.53(\mathrm{~s}$, $\left.3 \mathrm{H}, \mathrm{SCH}_{3}\right), 3.63\left(\mathrm{~s}, 2 \mathrm{H}, \mathrm{CH}_{2}\right)$ 7.30-7.20 (m, 4H, Ar-H). ${ }^{13} \mathrm{C}$ NMR (100 MHz, $\left.\mathrm{CDCl}_{3}, \delta, \mathrm{ppm}\right): 14.5,25.1,25.7,26.2,26.4,26.8,32.1$, $34.4,117.8,126.4,126.7,127.4,126.9,135.8,138.3,155.5$, 171.5, 174.6. MS (ESI, $m / z): 335.04\left(\mathrm{M}^{+}\right)$. Anal. calcd. for $\mathrm{C}_{18} \mathrm{H}_{22} \mathrm{O}_{4} \mathrm{~S}$ : C, 64.65; H, 6.63. Found: C, 64.63; H, 6.61\%.

2-(Cyclohexyl(4-fluorophenyl)methylene)succinic acid (4e): Color: Light yellow gummy mass. Yield: $92.94 \%$. IR (KBr, v, $\mathrm{cm}^{-1}$ ): 3450 (carboxylic OH), $1685\left(\mathrm{CH}_{2}-\mathrm{C}=0\right), 1662(\alpha, \beta$ unsaturated $\mathrm{C}=0$ ). ${ }^{1} \mathrm{H}$ NMR $\left(400 \mathrm{MHz}, \mathrm{CDCl}_{3}, \delta, \mathrm{ppm}\right): 0.93-1.65$ $\left(\mathrm{m}, 10 \mathrm{H}\right.$, cyclic $\left.-\mathrm{CH}_{2}\right), 2.27$ (p, $1 \mathrm{H}$, cyclic $\left.-\mathrm{CH}\right), 3.62\left(\mathrm{~s}, 2 \mathrm{H},-\mathrm{CH}_{2}\right)$, 6.84-7.30 (m, 4H, Ar-H). ${ }^{13} \mathrm{C}$ NMR (100 MHz, $\left.\mathrm{CDCl}_{3}, \delta, \mathrm{ppm}\right)$ : 25.1, 25.3, 25.3, 26, 26.3, 26.4, 32.5, 34.4, 115.1, 115.7, 117, 128.4, 128.9, 135.4, 155.1, 135.4, 155.1, 162.4, 171.4, 174.9. MS (ESI, $m / z$ ): $306.03\left(\mathrm{M}^{+}\right)$. Anal. calcd. for $\mathrm{C}_{17} \mathrm{H}_{19} \mathrm{FO}_{4}$ : C, 66.65; $\mathrm{H}$, 6.25. Found: $\mathrm{C}, 66.63 ; \mathrm{H}, 6.21 \%$.

\subsubsection{General procedure for the preparation of substituted 2-(cyclohexyl(phenyl)methyl)succinic acid (5a-e)}

In an autoclave vessel, substituted 2-cyclohexyl(phenyl methylenesuccinic acid (4a-e) (3 g, $0.0094 \mathrm{~mol}$ ) in methanol $(30 \mathrm{~mL})$ and $10 \% \mathrm{Pd} / \mathrm{C}(0.3 \mathrm{~g})$ were taken under nitrogen gas atmosphere. The reaction mixture was hydrogenated at 3-4 $\mathrm{kg} / \mathrm{cm}^{3}$ hydrogen pressure in the presence of palladium over carbon at $25-30{ }^{\circ} \mathrm{C}$ for $3-4 \mathrm{~h}$. The $\mathrm{Pd} / \mathrm{C}$ was filtered through hyflobed under nitrogen gas atmosphere. The mixture was concentrated under reduced pressure. The products were recrystallized from ethanol (Scheme 1).

2-(Cyclohexyl(4-methoxyphenyl)methyl)succinic acid (5a): Color: White solid. Yield: $78.21 \%$. M.p.: $152-154^{\circ} \mathrm{C}$. IR (KBr, $v$, $\left.\mathrm{cm}^{-1}\right): 3460$ (carboxylic $\left.\mathrm{OH}\right), 1708\left(\mathrm{CH}_{2}-\mathrm{C}=0\right), 1660(\alpha, \beta$ unsaturated $\mathrm{C}=0), 1590(\mathrm{Ar} \mathrm{C}=\mathrm{C}) .{ }^{1} \mathrm{H}$ NMR $\left(400 \mathrm{MHz}, \mathrm{CDCl}_{3}, \delta\right.$, ppm): 0.86-1.52 (m, 10H, cyclic - $\left.\mathrm{CH}_{2}\right), 1.80-2.24(\mathrm{~m}, 1 \mathrm{H}$, cyclic $\mathrm{CH}), 2.63\left(\mathrm{~d}, 2 \mathrm{H},-\mathrm{CH}_{2}\right), 3.06-3.38(\mathrm{~m}, 2 \mathrm{H}, \mathrm{CH}-\mathrm{CH}), 3.76(\mathrm{~s}, 3 \mathrm{H}$, $\left.\mathrm{OCH}_{3}\right)$, 6.80-7.23 (m, 4H, Ar-H). ${ }^{13} \mathrm{C}$ NMR $\left(100 \mathrm{MHz}, \mathrm{CDCl}_{3}, \delta\right.$, ppm): 26.2, 26.3, 26.6, 31.2, 31.4, 36.5, 41.7, 55.7, 114.2, 114.4, 129.4, 129.7, 133.7, 157.8, 173.4, 178.6. MS (ESI, $m / z): 321.16$ $\left(\mathrm{M}^{+}\right)$. Anal. calcd. for $\mathrm{C}_{18} \mathrm{H}_{24} \mathrm{O}_{5}$ : $\mathrm{C}, 67.48 ; \mathrm{H}, 7.55$. Found: $\mathrm{C}$, $67.47 ; \mathrm{H}, 7.52 \%$.

2-(Cyclohexyl(phenyl)methyl)succinic acid (5b): Color: White gummy mass. Yield: $78.4 \%$. IR $\left(\mathrm{KBr}, v, \mathrm{~cm}^{-1}\right): 3502$ (carboxylic $\mathrm{OH}), 1700\left(\mathrm{CH}_{2}-\mathrm{C}=0\right), 1658(\alpha, \beta$ unsaturated $\mathrm{C}=0)$. ${ }^{1} \mathrm{H}$ NMR $\left(400 \mathrm{MHz}, \mathrm{CDCl}_{3}, \delta, \mathrm{ppm}\right): 0.93-1.74(\mathrm{~m}, 10 \mathrm{H}$, cyclic $\left.\mathrm{CH}_{2}\right), 1.90-2.32(\mathrm{~m}, 1 \mathrm{H}$, cyclic $-\mathrm{CH}), 3.64\left(\mathrm{~d}, 2 \mathrm{H},-\mathrm{CH}_{2}\right), 3.10-3.30$ (m, 2H, CH-CH), 6.90-7.30 (m, 5H, Ar-H). ${ }^{13} \mathrm{C}$ NMR (100 MHz, $\left.\mathrm{CDCl}_{3}, \delta, \mathrm{ppm}\right): 26.3,26.4,26.8,30.2,31.1,31.9,36.9,37.4,41.4$, $125.9,128.1,128.2,128.3,128.4,141.3,173.8,178.4,173.8$, 178.4. MS (ESI, $m / z$ ): $291.10\left(\mathrm{M}^{+}\right)$. Anal. calcd. for $\mathrm{C}_{17} \mathrm{H}_{22} \mathrm{O}_{4}$ : C, 70.32; H, 7.64. Found: $\mathrm{C}, 70.31 ; \mathrm{H}, 7.61 \%$.

2-(Cyclohexyl(p-tolyl)methyl)succinic acid (5c): Color: White solid. Yield: 96.47 \%. M.p.: $180-183{ }^{\circ} \mathrm{C}$. IR (KBr, v, cm-1 $): 3440$ (carboxylic $\mathrm{OH}), 1709\left(\mathrm{CH}_{2}-\mathrm{C}=0\right), 1666(\alpha, \beta$ unsaturated $\mathrm{C}=0)$, 
2920 (methyl CH). ${ }^{1} \mathrm{H}$ NMR $\left(400 \mathrm{MHz}, \mathrm{CDCl}_{3}, \delta, \mathrm{ppm}\right): 1.10-1.40$ $\left(\mathrm{m}, 10 \mathrm{H}\right.$, cyclic $\left.-\mathrm{CH}_{2}\right), 1.83-2.27(\mathrm{~m}, 1 \mathrm{H}$, cyclic $-\mathrm{CH}), 2.54(\mathrm{~s}, 3 \mathrm{H},-$ $\left.\mathrm{CH}_{3}\right), 3.68\left(\mathrm{~d}, 2 \mathrm{H},-\mathrm{CH}_{2}\right), 3.03-3.32(\mathrm{~m}, 2 \mathrm{H}, \mathrm{CH}-\mathrm{CH}), 6.93-7.44$ (m, $4 \mathrm{H}, \mathrm{Ar}-\mathrm{H}) .{ }^{13} \mathrm{C}$ NMR $\left(100 \mathrm{MHz}, \mathrm{CDCl}_{3}, \delta, \mathrm{ppm}\right): 178.3,173.4$, 138.6, 135.4, 128.9, 128.7, 128.4, 128.1, 41.7, 37.4, 36.5, 31.4, 31.2, 30.0, 26.4, 26.3, 26.1, 21.6. MS (ESI, $\mathrm{m} / z$ ): $305.09\left(\mathrm{M}^{+}\right)$. Anal. calcd. for $\mathrm{C}_{18} \mathrm{H}_{24} \mathrm{O}_{4}$ : C, 71.03; H, 7.95. Found: C, 71.00; $\mathrm{H}$, $7.92 \%$.

2-(Cyclohexyl(4-(methylthio)phenyl)methyl)succinic acid (5d): Color: White gummy mass. Yield: $73.89 \%$. IR (KBr, $v, \mathrm{~cm}^{-}$ 1): 3405 (carboxylic $\mathrm{OH}), 1692\left(\mathrm{CH}_{2}-\mathrm{C}=0\right), 1663(\alpha, \beta$ unsaturated $\mathrm{C}=0) .{ }^{1} \mathrm{H}$ NMR $\left(400 \mathrm{MHz}, \mathrm{CDCl}_{3}, \delta, \mathrm{ppm}\right): 0.83-1.45$ (m, 10H, cyclic $\mathrm{CH}_{2}$ ), 1.80-2.36 (m, $1 \mathrm{H}$, cyclic $\left.-\mathrm{CH}\right), 2.56(\mathrm{~s}, 3 \mathrm{H}$, $\left.\mathrm{SCH}_{3}\right), 3.62$ (d, $2 \mathrm{H},-\mathrm{CH}_{2}$ ), 3.10-3.46 (m, $\left.2 \mathrm{H}, \mathrm{CH}-\mathrm{CH}\right), 7.16-7.47$ (m, 4H, Ar-H). ${ }^{13} \mathrm{C}$ NMR $\left(100 \mathrm{MHz}, \mathrm{CDCl}_{3}, \delta, \mathrm{ppm}\right): 21.1,26.6$, 26.4, 30, 31.2, 31.4, 36.5, 37.4, 41.7, 128.1, 128.4, 128.7, 128.9, 135.4, 138.6, 173.4, 178.9. MS (ESI, $m / z): 337.01\left(\mathrm{M}^{+}\right)$. Anal. calcd. for $\mathrm{C}_{18} \mathrm{H}_{24} \mathrm{O}_{4} \mathrm{~S}$ : C, 64.26; H, 7.19. Found: C, 64.24; $\mathrm{H}$, 7.15\%.

2-(Cyclohexyl(4-fluorophenyl)methyl)succinic acid (5e): Color: White gummy mass. Yield: $75.35 \%$. IR $\left(\mathrm{KBr}, v, \mathrm{~cm}^{-1}\right)$ : 3495 (carboxylic $\mathrm{OH}), 1708\left(\mathrm{CH}_{2}-\mathrm{C}=0\right), 1664(\alpha, \beta$ unsaturated $\mathrm{C}=0) .{ }^{1} \mathrm{H}$ NMR $\left(400 \mathrm{MHz}, \mathrm{CDCl}_{3}, \delta, \mathrm{ppm}\right): 0.83-1.49(\mathrm{~m}, 10 \mathrm{H}$, cyclic $\left.-\mathrm{CH}_{2}\right), 1.86-2.23(\mathrm{~m}, 1 \mathrm{H}$, cyclic $-\mathrm{CH}), 3.53\left(\mathrm{~d}, 2 \mathrm{H},-\mathrm{CH}_{2}\right)$, 3.11-3.34 (m, 2H, CH-CH), 6.90-7.36 (m, 4H, Ar-H). ${ }^{13} \mathrm{C}$ NMR (100 MHz, $\left.\mathrm{CDCl}_{3}, \delta, \mathrm{ppm}\right): 14.2,26.1,26.4,26.7,30.4,31.3,31.6$, 36.4, 37.4, 41.5, 128, 128.2, 128.4, 128.8, 138.4, 139.4, 178.9 . MS (ESI, $m / z$ ): $309.11\left(\mathrm{M}^{+}\right)$. Anal. calcd. for $\mathrm{C}_{17} \mathrm{H}_{21} \mathrm{FO}_{4}$ : C, 66.22; H, 6.86. Found: C, 66.19; H, 6.82\%.

\subsubsection{General procedure for the preparation of 1-cyclohexyl- 4-oxo-1,2,3,4-tetrahydronaphthalene-2-carboxylic acid (7a-} e)

Substituted 2-(cyclohexyl(phenyl)methyl)succinic acid (5ae) $(3 \mathrm{~g}, 0.00937 \mathrm{~mol})$ and acetyl chloride $(30 \mathrm{~mL})$ mixture was refluxed for $6 \mathrm{~h}$. The completion of the reaction was known by TLC. The excess of the acetyl chloride was distilled off under atmospheric pressure. The resultant mixture was dissolved in dichloromethane $(25 \mathrm{~mL})$ and washed with $5 \%$ cold sodium bicarbonate solution. The separated organic layer was finally washed with water and dried over anhyd sodium sulphate. Distilled off solvent to afford a gummy mass (6a-e) in good yield (Scheme 1).

Above compounds (6a-e) $(1 \mathrm{~g}, 0.0033 \mathrm{~mol})$ were dissolved in dichloromethane $(10 \mathrm{~mL})$ and added dropwise to the stirred suspensions of anhydride aluminum chloride $(1.1 \mathrm{~g}, 0.0082$ $\mathrm{mol})$ in dichloromethane $(10 \mathrm{~mL})$ at $0-5{ }^{\circ} \mathrm{C}$ under nitrogen gas atmosphere. The reaction mixture was stirred for $6 \mathrm{~h}$ at 25-30 ${ }^{\circ} \mathrm{C}$. The completion of the reaction was known by TLC. The reaction mass was poured into $1 \mathrm{~N} \mathrm{HCl}(30 \mathrm{~mL})$. The product was extracted into dichloromethane and then into saturated $\mathrm{Na}_{2} \mathrm{CO}_{3}$ solution. The sodium carbonate extracts were acidified by dil. $\mathrm{HCl}$ to give products in good yields. They were recrystallized from ethanol (Scheme 1).

1-Cyclohexyl-6-methoxy-4-oxo-1,2,3,4-tetrahydro naphthalene-2-carboxylic acid (7a): Color: Yellow solid. Yield: $66.18 \%$. M.p.: $199-201^{\circ} \mathrm{C}$. IR (KBr, v, cm-1): 3396 (carboxylic OH), 1715 (carboxylic $\mathrm{C}=0), 1702$ (tetralone $\mathrm{C}=0), 1597(\mathrm{Ar} \mathrm{C}=\mathrm{C}) .{ }^{1} \mathrm{H}$ NMR ( $\left.400 \mathrm{MHz}, \mathrm{CDCl}_{3}, \delta, \mathrm{ppm}\right): 0.89-1.30\left(\mathrm{~m}, 10 \mathrm{H}\right.$, cyclic $\left.-\mathrm{CH}_{2}\right), 1.43-$ $1.76(\mathrm{~m}, 1 \mathrm{H}$, cyclic $-\mathrm{CH}), 2.37\left(\mathrm{dd}, 2 \mathrm{H},-\mathrm{CH}_{2}\right), 3.02(\mathrm{t}, 1 \mathrm{H}, \mathrm{CH})$, 3.46 (q, 1H, -CH), 3.78 (s, 3H, $\left.-\mathrm{OCH}_{3}\right), 7.10-7.44(\mathrm{~m}, 3 \mathrm{H}, \mathrm{Ar}-\mathrm{H})$. ${ }^{13} \mathrm{C}$ NMR $\left(100 \mathrm{MHz}, \mathrm{CDCl}_{3}, \delta, \mathrm{ppm}\right): 26.0,26.1,26.4,31.2,36.4$, 37.5, 39.4, 55.3, 108.8, 122.8, 129.3, 130.4, 134.7, 157.6, 178.5, 196.4. MS (ESI, $m / z): 303.12\left(\mathrm{M}^{+}\right)$. Anal. calcd. for $\mathrm{C}_{18} \mathrm{H}_{22} \mathrm{O}_{4}$ : C, 71.50; H, 7.33. Found: C, 71.47; H, 7.31\%.

1-Cyclohexyl-4-oxo-1,2,3,4-tetrahydronaphthalene-2-carbo xylic acid (7b): Color: Yellow solid. Yield: $64.90 \%$. M.p.: 170$172{ }^{\circ} \mathrm{C}$. IR (KBr, $v, \mathrm{~cm}^{-1}$ ): 3435 (carboxylic OH), 1701 (tetralone $\mathrm{C}=0), 1716$ (carboxylic $\mathrm{C}=0), 1595(\mathrm{Ar} \mathrm{C}=\mathrm{C}) .{ }^{1} \mathrm{H}$ NMR $(400 \mathrm{MHz}$, $\left.\mathrm{CDCl}_{3}, \delta, \mathrm{ppm}\right)$ : 0.93-1.68 (m, 10H, cyclic $\left.-\mathrm{CH}_{2}\right), 1.75-1.97(\mathrm{~m}$,
$1 \mathrm{H}$, cyclic - $\mathrm{CH}$ ), 2.37 (dd, $\left.2 \mathrm{H},-\mathrm{CH}_{2}\right), 2.87(\mathrm{t}, 1 \mathrm{H},-\mathrm{CH}), 3.23-3.47$ (q, 1H, CH), 6.87-7.43 (m, 4H, Ar-H). ${ }^{13} \mathrm{C}$ NMR $\left(100 \mathrm{MHz} \mathrm{CDCl}_{3}\right.$, $\delta$, ppm): 26.2, 26.3, 26.5, 31.5, 31.7, 31.3, 36.5, 37.5, 38.4, 39.7, 123.8, 125.6, 127.2, 131.6, 132.4, 138.5, 178.4, 196.5. MS (ESI, $m / z): 272.05\left(\mathrm{M}^{+}\right)$. Anal. calcd. for $\mathrm{C}_{17} \mathrm{H}_{20} \mathrm{O}_{3}$ : C, 74.97; $\mathrm{H}, 7.40$. Found: C, 74.93; H, 7.37\%.

1-Cyclohexyl-6-methyl-4-oxo-1,2,3,4-tetrahydronaphthalene2-carboxylic acid (7c): Color: Yellow solid. Yield: $61.81 \%$. M.p.: 184-186 ${ }^{\circ} \mathrm{C}$. IR $\left(\mathrm{KBr}, v, \mathrm{~cm}^{-1}\right): 3435$ (carboxylic OH), 1705 (tetralone $\mathrm{C}=0$ ), 1718 (carboxyl $\mathrm{C}=0$ ), 1714 (carboxylic $\mathrm{C}=0$ ), 1594 ( $\mathrm{Ar} \mathrm{C}=\mathrm{C}) .{ }^{1} \mathrm{H}$ NMR $\left(400 \mathrm{MHz}, \mathrm{CDCl}_{3}, \delta, \mathrm{ppm}\right): 1.13-1.53$ (m, $10 \mathrm{H}$, cyclic $-\mathrm{CH}_{2}$ ), 1.80-2.17 (m, $1 \mathrm{H}$, cyclic $\left.-\mathrm{CH}\right), 2.32(\mathrm{~s}, 3 \mathrm{H},-$ $\left.\mathrm{CH}_{3}\right), 2.50\left(\mathrm{dd}, 2 \mathrm{H}, \mathrm{CH}_{2}\right), 2.93-3.24(\mathrm{t}, 1 \mathrm{H}, \mathrm{CH}), 3.67-3.83(\mathrm{q}, 1 \mathrm{H}$, $\mathrm{CH})$, 6.86-7.42 (m, 3H, Ar-H). ${ }^{13} \mathrm{C}$ NMR $\left(100 \mathrm{MHz}, \mathrm{CDCl}_{3}, \delta\right.$, ppm): $21.8,26.2,26.3,26.4,31.2,31.6,36.5,37.6,38.7,39.5$, 124.7, 127.6, 133.5, 135.4, 135.6, 140.5, 178.6, 196.8. MS (ESI, $m / z): 287.06\left(\mathrm{M}^{+}\right)$. Anal. calcd. for $\mathrm{C}_{18} \mathrm{H}_{22} \mathrm{O}_{3}$ : C, 75.50; $\mathrm{H}, 7.74$. Found: C, 75.48; H, 7.71\%.

1-Cyclohexyl-6-(methylthio)-4-oxo-1,2,3,4-tetrahydro naphthalene-2-carboxylic acid (7d): Color: Yellow solid. Yield: 67.72 \%. M.p.: $161-163{ }^{\circ} \mathrm{C}$. IR $\left(\mathrm{KBr}, v, \mathrm{~cm}^{-1}\right)$ : 3440 (carboxylic $\mathrm{OH}$ ), 1710 (tetralone $\mathrm{C}=0$ ), 1718 (carboxyl $\mathrm{C}=0$ ), $1587\left(\mathrm{Ar} \mathrm{C}=\mathrm{C}\right.$ ). ${ }^{1} \mathrm{H}$ NMR (400 MHz, $\left.\mathrm{CDCl}_{3}, \delta, \mathrm{ppm}\right): 1.13-1.44\left(\mathrm{~m}, 10 \mathrm{H}\right.$, cyclic $\left.-\mathrm{CH}_{2}\right)$, 1.86-2.18 (m, $1 \mathrm{H}$, cyclic - $\mathrm{CH}), 2.49\left(\mathrm{~s}, 3 \mathrm{H}, \mathrm{SCH}_{3}\right), 2.61(\mathrm{dd}, 2 \mathrm{H}$, $\mathrm{CH}_{2}$ ), $3.07(\mathrm{t}, 1 \mathrm{H}, \mathrm{CH}), 3.53-3.72\left(\mathrm{q}, 1 \mathrm{H},-\mathrm{CH}_{2}\right), 7.06-7.48(\mathrm{~m}, 3 \mathrm{H}$, $\mathrm{Ar}-\mathrm{H}) .{ }^{13} \mathrm{C}$ NMR (100 MHz, $\left.\mathrm{CDCl}_{3}, \delta, \mathrm{ppm}\right): 14.3,26.3,26.4,26.8$, $31.3,31.5,36.4,37.6,38.4,39.5,124.3,128.9,132.8,134.7$ 135.9, 136.7, 178.7, 196.5. MS (ESI, $m / z): 319.03\left(\mathrm{M}^{+}\right.$). Anal. calcd. for $\mathrm{C}_{18} \mathrm{H}_{22} \mathrm{O}_{3} \mathrm{~S}: \mathrm{C}, 67.89 ; \mathrm{H}, 6.96$. Found: C, 67.87; $\mathrm{H}$, $6.92 \%$.

1-Cyclohexyl-6-fluoro-4-oxo-1,2,3,4-tetrahydronaphthalene2-carboxylic acid (5e): Color: Yellow solid. Yield: $66.94 \%$. M.p.: 201-203 ${ }^{\circ} \mathrm{C}$. IR (KBr, v, cm-1): 3390 (carboxylic OH), 1703 (tetralone $\mathrm{C}=0$ ), 1720 (carboxyl $\mathrm{C}=0$ ), $1599(\mathrm{Ar} \mathrm{C}=\mathrm{C}) .{ }^{1} \mathrm{H}$ NMR ( $\left.400 \mathrm{MHz}, \mathrm{CDCl}_{3}, \delta, \mathrm{ppm}\right): 0.96-1.46\left(\mathrm{~m}, 10 \mathrm{H}\right.$, cyclic $\left.-\mathrm{CH}_{2}\right), 1.63$ $1.85(\mathrm{~m}, 1 \mathrm{H}$, cyclic $-\mathrm{CH}), 2.42\left(\mathrm{dd}, 2 \mathrm{H}, \mathrm{CH}_{2}\right), 2.83-3.15(\mathrm{t}, 1 \mathrm{H}$, $\mathrm{CH}), 3.42-3.68(\mathrm{q}, 1 \mathrm{H}, \mathrm{CH}), 7.18-7.69(\mathrm{~m}, 3 \mathrm{H}, \mathrm{Ar}-\mathrm{H}) .{ }^{13} \mathrm{C}$ NMR $\left(100 \mathrm{MHz}, \mathrm{CDCl}_{3}, \delta, \mathrm{ppm}\right): 26.0,26.3,26.7,31.4,31.8,36.1,37.5$, 38.6, 39.4, 113.4, 119.9, 129.4, 134.6, 135.4, 160.6, 178.8, 196.5 MS (ESI, $m / z$ ): $291.01\left(\mathrm{M}^{+}\right)$. Anal. calcd. for $\mathrm{C}_{17} \mathrm{H}_{19} \mathrm{FO}_{3}$ : C, 70.33; $\mathrm{H}, 6.60$. Found: $\mathrm{C}, 70.30 ; \mathrm{H}, 6.57 \%$.

\subsection{Antimitotic study}

The novel tetralone acid analogues of podophyllotoxin (7ae) were screened for antimitotic activity by onion root tip method and the $\mathrm{ID}_{50}$ was determined. The materials required are acetoorcein solution, compound microscope, glass slides, cover slips, hydrochloric acid ( $0.1 \mathrm{~N})$, Carney's solution II, 70\% ethanol and tested samples (100, 200 and $\left.400 \times 10^{-6} \mathrm{~mol} / \mathrm{L}\right)$. To study the effect of novel tetralone acids analogues of podophyllotoxin on somatic cells, onion base was immersed to an extent of about half a centimeter in a sample tube and control solution tube $(7 \times 3)$, after removing the old roots from them and immersion is continued for $24 \mathrm{~h}$. for germination. After different time intervals, the germinated root tips were removed and were fixed in Carney's solution II (alcohol and acetic acid in 3:1 ratio respectively) for $24 \mathrm{~h}$. After $24 \mathrm{~h}$. Carney's solution II was decanted carefully and the root tips were washed with preserving solvent $(70 \%$ ethanol). The fixed root tips were preserved in $70 \%$ ethanol in refrigerator. The root tips were taken in watch glass and stained with a drop of acetoorcein stain and a drop of $1 \mathrm{~N} \mathrm{HCl}(7: 1, v: v)$. The glasses were warmed and kept for $1 \mathrm{hr}$. The roots were taken on a clean glass slide and squashed using $45 \%$ acetic acid following the method of Levan [11]. A microscope cover glass was placed on the material and then pressure was applied on a cover glass to ensure uniform spreading. The cover glass was sealed with molten paraffin wax and slide was observed under microscope. 
Table 1. Antimitotic activity of the compounds (7a-e) by onion root tip method.

\begin{tabular}{|c|c|c|c|c|c|}
\hline Compound & $\begin{array}{l}\text { Concentration } \\
\left(\times 10^{-6} \mathrm{~mol} / \mathrm{L}\right)\end{array}$ & \% Dividing Cell & $\begin{array}{l}\% \text { Dividing cells } \\
\text { compared to control }\end{array}$ & $\begin{array}{l}\text { \% Dividing inhibition } \\
\text { compared to control }\end{array}$ & $\begin{array}{l}\mathrm{IC}_{50} \\
\left(\times 10^{-6} \mathrm{~mol} / \mathrm{L}\right)\end{array}$ \\
\hline \multirow[t]{3}{*}{$7 a$} & 2.03 & 7.513 & 35.57 & 64.43 & \multirow[t]{3}{*}{1.66} \\
\hline & 3.02 & 5.981 & 28.32 & 71.68 & \\
\hline & 4.01 & 4.736 & 22.42 & 77.58 & \\
\hline \multirow[t]{3}{*}{$7 \mathrm{~b}$} & 2.03 & 13.01 & 61.60 & 38.40 & \multirow[t]{3}{*}{9.12} \\
\hline & 3.04 & 13.45 & 63.68 & 36.32 & \\
\hline & 4.02 & 13.87 & 65.67 & 34.33 & \\
\hline \multirow[t]{3}{*}{$\overline{7 c}$} & 2.01 & 11.06 & 52.37 & 47.63 & \multirow[t]{3}{*}{6.42} \\
\hline & 3.03 & 10.68 & 50.57 & 49.43 & \\
\hline & 4.04 & 10.21 & 48.34 & 51.66 & \\
\hline \multirow[t]{3}{*}{$\overline{7 d}$} & 2.03 & 06.34 & 30.02 & 69.98 & \multirow[t]{3}{*}{1.58} \\
\hline & 3.04 & 05.12 & 24.24 & 75.76 & \\
\hline & 4.02 & 04.06 & 19.22 & 80.78 & \\
\hline \multirow[t]{3}{*}{$7 e$} & 2.01 & 08.06 & 38.16 & 61.84 & \multirow[t]{3}{*}{5.83} \\
\hline & 3.02 & 08.67 & 41.05 & 58.95 & \\
\hline & 4.05 & 08.01 & 37.93 & 62.07 & \\
\hline Control & - & 21.12 & 100 & 00.00 & 0.00 \\
\hline
\end{tabular}

Mitotic Index (MI) was calculated by following method of Fissceja [12]. The mitotic index was determined by examination of minimum of zone cells. Three replicates were made for each calculation. The slides were observed under microscope and photographed.

M. I. $=\frac{\text { Total number of dividing cells }}{\text { Total number of cells examined }} \times 100$

The percentage of the number of dividing cells compared to the control and the percent inhibition of mitosis by antimitotic agent at a different concentration such as 100, 200, and $400 \times 10^{-6} \mathrm{~mol} / \mathrm{L}$ against a control were calculated. The concentration needed for $50 \%$ inhibition ( $\left.\mathrm{ID}_{50}\right)$ was extrapolated from the graph of the concentration verses percentage inhibition. ID 50 values for novel tetralone acid analogues of podophyllotoxin for antimitotic activity were calculated individually following hakala [13] method.

\section{Results and discussion}

\subsection{Chemistry}

The tetralone acid analogues of podophyllotoxin were synthesized by gensler's method (Scheme 1). Substituted cyclohexyl(phenyl)methanone (2a-e) were prepared in high yields by stirring an equimolar solution of substituted benzenes separately with cyclohexyl carbonyl chloride in the presence of anhyd. aluminium chloride in dichloromethane [14]. Itaconic acid half esters (3a-e) were prepared as a mixture of cis and trans isomers by Stobbe condensation of substituted cyclohexyl (phenyl)methanone (2a-e) with diethyl succinate using sodium hydride as a base in benzene and absolute ethanol at room temperature [15]. Substituted 2-(cyclohexyl(phenyl)methylene)succinic acid (4a-e) were prepared by the saponification of itaconic acid half esters by refluxing in methanol and water mixture in the presence of sodium hydroxide. Substituted 2(cyclohexyl(phenyl)methyl)succinic acid (5a-e) were prepared by the catalytic hydrogenation in the presence of palladium over carbon in methanol under $3-4 \mathrm{~kg} / \mathrm{cm}^{3}$ hydrogen pressure at $25-30{ }^{\circ} \mathrm{C}[16,17]$. Benzhydryl succinic anhydrides (6a-e) were prepared by refluxing benzhydryl succinic acids with acetyl chloride. Tetralone acids (7a-e) were prepared by the intramolcular Friedel-Craft's acylation reaction of benzhydryl succinic anhydrides in the presence of Lewis acids anhyd. aluminium chloride in dichloromethane (Scheme 1) [18,19]. The products were characterized by IR, ${ }^{1} \mathrm{H}$ NMR, ${ }^{13} \mathrm{C}$ NMR, mass spectral data and elemental analysis data.

Tetralone acids $\mathbf{7 a}$ and $\mathbf{7 d}$ were obtained in good yields (65-68\%). The products $7 \mathbf{b}, \mathbf{7 c}$ and $7 \mathrm{e}$ were formed in moderate yields (50-53\%). The formation of moderate yield might be due to the less electron donating nature of hydrogen, methyl and fluorine linked phenyl ring. Hence the $\pi$-electrons of benzene ring are not readily available for Friedel-Craft's intramaolecular acylation reactions. The NMR spectrum of tetralone acid 7a showed a triplet at 3.3-3.6 ppm $(J=6 \mathrm{~Hz}$ ) for the $\mathrm{C}_{1}-\mathrm{H}$. The large coupling constant $(J)$ value indicated that $\mathrm{C}_{1}-\mathrm{H}$ and $\mathrm{C}_{2}-\mathrm{H}$ in 7a were diaxial. Hence the $\mathrm{C}_{2}-\mathrm{H}$ carbonyl and $\mathrm{C}_{1}-\mathrm{H}$ cyclohexyl groups should be trans to each other, a configuration being thermodynamically more stable.

\subsection{Antimitotic activity}

As regards the relationships between the structure of the podophyllotoxin scaffold and antimitotic properties, it showed varied antimitotic activity (Table 1). The presence of different substituents on the ring A causes a certain changes in activity. The compound $\mathbf{7 b}$ has hydrogen, moiety on ring $\mathrm{A}$, which is accounted for the enhanced antimitotic activity than when compared to control solution. Similarly compounds $\mathbf{7 c}$ and $\mathbf{7 e}$ have showed significant activity. The compounds $\mathbf{7 c}$ and $\mathbf{7 e}$ have methyl and fluorine moiety on ring $\mathrm{A}$, which is accounted for the moderate activity of the compounds. On the other hand, the remaining compounds $\mathbf{7 a}$ and $\mathbf{7 d}$ have showed less activity compared to control. From the obtained results, it is clear that the major role for antimitotic activity is played by substituents on ring A moiety. It is evident that novel tetralone acid analogues of podophyllotoxin were showed good antimitotic activity.

\section{Conclusion}

In conclusion, the new tetralone acid analogues of podophyllotoxin (7a-e) were synthesized in good yields following Gensler's method with some changes in reagents and reaction conditions. The structures of synthesized compounds were confirmed and characterized by analytical data's such as IR, ${ }^{1} \mathrm{H}$ NMR, ${ }^{13} \mathrm{C}$ NMR, Mass spectra and elemental analysis. They were screened for antimitotic activity. The novel tetralone acids analogues of podophyllotoxin were synthesized by replacing methylene dioxy group in podophyllotoxin with methoxy, methyl, thiomethyl group and hydrogen and fluorine and the trimethoxyphenyl ring with cyclohexyl and lactone ring with carboxylic acid to study the structure activity relationship. It is noteworthy that compound $\mathbf{7 b}$ possessed good antimitotic activity, 7c and 7e showed considerable activity and remaining $\mathbf{7 a}$ and $\mathbf{7 d}$ possessed low activity.

\section{Acknowledgement}

The authors are thankful to University of Mysore, Mysore for providing spectral data. 


\section{References}

[1]. Podwyssotzki, V. Arch. Expt. Path. U. Pharmakol. 1880, 13, 29-52.

[2]. Jardine, I. Anticancer Agents Based on Natural Product Models, Cassady, J. M.; Douros, J. D. Eds., Academic Press, New York, 1980, pp. 319-351.

[3]. Hartwell, J. L.; Schrecker, A. W. Fortschr. Chem. Org. Naturst. 1958, 13, 83-166.

[4]. Smissman, E. E.; Murray, R. J.; McChesney, J. D.; Houston, L. L.; Pazdernik, T. L. J. Med. Chem. 1976, 19, 148-153.

[5]. Schreir, E. A. 152nd National Meeting of the American Chemical Society, New York, 1966, pp. 34.

[6]. Anjanamurthy, C.; Lokanatha Rai, K. M. Indian J. Chem. Sect. B. 1987, 26, 131-135.

[7]. Ward, R. S. Chem. Soc. Rev. 1982, 11, 003-004.

[8]. Basavaraju, Y. B.; Anjanamurthy, C. Indian J. Chem. Sect. B. 2003, 42, 876-880.

[9]. Gensler, W. J.; Samour, C. M.; Wand, S. Y.; Johnson, F. J. Am. Chem. Soc. 1960, 82, 1714-1727.

[10]. Lokanatha, R. K. M.; Basavaraju, Y. B.; Sadashivamurthy, B. Indian J. Pharm. Sci. 2007, 69, 116-118.

[11]. Fissceja, G. Hereditas. 1985, 102, 099-112.

[12]. Levan, A. Hereditas. 1938, 24, 471-486.

[13]. Hakala, T. R.; Lange, P. A.; Fraley, E. F. Estimation of Human Cell Mediated Cytotoxicity by Lymphocyte Titration and Automated Image Analysis. In: In Vitro Methods in Cell-Mediated Immunity, VII, Bloom, B. R.; David, J. R., Eds., Academic Press, New York, 1976, pp. 451-460.

[14]. Hemakumar, K. H.; Sathisha, A. D.; Basavaraju, Y. B. E-J. Chem. 2008, 5, 114-119.

[15]. Daub, G. H.; Johnso, W. S. The Stobbe Condensation with Sodium Hydride, Organic reactions, John Wiley and Sons, New York, 1950, pp. 501-504.

[16]. Siegel, S.; Dunkel, M.; Smith, G. V.; Halpern, W.; Cozort, J. J. Org. Chem. 1966, 31, 2802-2806.

[17]. Gault, F. G.; Rooney, J. J.; Kemball, C. J. Catal. 1962, 1, 255-274.

[18]. Gensler, W. J.; Murthy, C. D.; Trammell M. H. J. Med. Chem. 1977, 20 , 635-644.

[19]. Gensler, W. J.; Gatsonis, C. D. J. Med. Chem. 1966, 31, 4004-4008. 